\title{
Endoscopic Submucosal Dissection Using a Novel Versatile Knife: An Animal Feasibility Study (with Video)
}

\author{
Chang-II Kwon', Gwangil Kim², II-Kwun Chung³, Won Hee Kim¹, Kwang Hyun Ko', Sung Pyo Hong', \\ Seok Jeong ${ }^{4}$ and Don Haeng Lee ${ }^{4}$ \\ ${ }^{1}$ Digestive Disease Center, ${ }^{2}$ Department of Pathology, CHA Bundang Medical Center, CHA University, Seongnam, ${ }^{3}$ Division of Gastroenterology \\ and Hepatology, Department of Internal Medicine, Soonchunhyang University Cheonan Hospital, Soonchunhyang University College of Medicine, \\ Cheonan, ${ }^{4}$ Division of Gastroenterology, Department of Internal Medicine, Inha University School of Medicine, Incheon, Korea
}

\begin{abstract}
Background/Aims: In order to reduce the procedure time and the number of accessory changes during endoscopic submucosal dissection (ESD), we developed a novel versatile knife, which has the combined advantages of several conventional knives. The aim of this study was to compare the efficacy, safety, and histological quality of ESD performed using this novel versatile knife and a combination of several conventional knives.

Methods: This was an in vivo animal study comparing two different modalities of ESD in mini-pigs. Completion time of each resection was documented, and the resected specimens were retrieved and evaluated for completeness. To assess the quality control of the procedures and adverse events, detailed histopathological examinations were performed.

Results: A total of 18 specimens were dissected by ESD safely and easily (nine specimens using the new versatile knife; nine specimens by mixing conventional knives). All resections were completed as en bloc resections. There was no significant difference in procedure time between the 2 modalities ( 456 seconds vs. 355 seconds, $p=0.258)$ and cutting speed $\left(1.983 \mathrm{~mm}^{2} / \mathrm{sec} v \mathrm{vs} .1 .57 \mathrm{~mm} / \mathrm{sec}, p=1.000\right)$. The rate of adverse events and histological quality did not statistically differ between the modalities.
\end{abstract}

Conclusions: ESD with a versatile knife appeared to be an easy, safe, and technically efficient method.

Key Words: Equipment and supplies; Endoscopic submucosal dissection; Models, animal; Instrumentation

\section{INTRODUCTION}

The development of endoscopic submucosal dissection (ESD) has allowed en bloc resection for accurate histological assessment and resection of tumors regardless of their sizes. ${ }^{1-8}$ Although ESD allows reliable resection of a large lesion en bloc, ESD with a knife is technically difficult, and requires special skills and a long procedure time for a quality-controlled Received: February 9, 2014 Revised: May 1, 2014

Accepted: June 12, 2014

Correspondence: Il-Kwun Chung

Division of Gastroenterology and Hepatology, Department of Internal Medicine, Soonchunhyang University Cheonan Hospital, Soonchunhyang University College of Medicine, 31 Suncheonhyang 6-gil, Dongnam-gu, Cheonan 330-930, Korea

Tel: +82-41-570-3679, Fax: +82-41-574-5762, E-mail: euschung@schmc.ac.kr Chang-Il Kwon and Gwang-Il Kim contributed equally to this work as first authors.

(c) This is an Open Access article distributed under the terms of the Creative Commons Attribution Non-Commercial License (http://creativecommons.org/ licenses/by-nc/3.0) which permits unrestricted non-commercial use, distribution, and reproduction in any medium, provided the original work is properly cited. procedure. In particular, gastric ESD in specific situations and esophageal and colonic ESDs are considered to be more difficult. ${ }^{5,9,10}$ To overcome these limitations and decrease the rate of procedural adverse events, different devices and several innovations have been introduced to improve technical outcomes. ${ }^{11-14}$

Despite improvements in equipment and ESD techniques, each ESD step may require different accessories or knives depending on the location and shape of the tumor to achieve quality-controlled ESD., ${ }^{9,15}$ Therefore, the accessories may need to be changed frequently due to the use of various accessories, which is time-consuming and expensive. Although there may be differences between countries, the ever-increasing use of ESD procedures is becoming a national burden that has to be covered with limited insurance benefits and is even more burdensome for patients and physicians if insurance is not available.

For reducing the need to change accessories and procedure time, we developed a novel versatile knife (Optimos knife; Tae- 
woong Medical, Goyang, Korea), which has the combined advantages of several conventional knives. The Optimos knife is capable of performing all steps in ESD in addition to water injection, and has several distinguishing features. The Optimos knife may enable endoscopists to perform quality-controlled ESD and reduce the procedure time and procedurerelated adverse events.

The aim of this study was to compare the efficacy and safety of the Optimos knife in ESD with those of several conventional knives.

\section{MATERIALS AND METHODS}

This was an in vivo animal study comparing two different modalities (Optimos knife and a combination of several conventional knives) for the removal of target mucosal lesions. This study was approved by the Institutional Animal Care and Use Committee of the Inha University School of Medicine.

\section{Animals}

This animal study was conducted at the National Center of Efficacy Evaluation for the Development of Health Products Targeting Digestive Disorders (Pyeongtaek, Korea), an ani- mal research institution. We used two male miniature pigs (Sus scrofa; Micropig, Hybrid; Yukatan, Vietnam potbelly) that weighed 31 and $33 \mathrm{~kg}$. After 2 days of fasting, the miniature pigs underwent ESD with general anesthesia and mechanical ventilation. Preanesthetic sedation consisted of an intramuscular injection of atropine sulfate $(0.04 \mathrm{mg} / \mathrm{kg})$, xylazine ( $2 \mathrm{mg} / \mathrm{kg})$, and tiletamine-zolazepam ( $5 \mathrm{mg} / \mathrm{kg})$. The animals were intubated and general anesthesia including $0.5 \%$ to $2 \%$ isoflurane via an endotracheal tube and $70 \%$ nitrous oxide and $30 \%$ oxygen provided by a ventilator was administered. The miniature pigs were then placed on their sides on an operation table. Cardiopulmonary parameters were monitored throughout the procedure.

\section{Newly developed Optimos knife}

The Optimos knife (Fig. 1, Supplementary Video 1 [available online at http://www.e-ce.org/]) is capable of performing several steps of ESD (including marking, circumferential incision and cutting, dissection, vessel coagulation, and water injection) without the need to change the instrument. The Optimos knife also has several other distinguishing functions. The tip of the knife can be rotated fully and smoothly by adjusting the dial on the handle. The transparent sheath of the
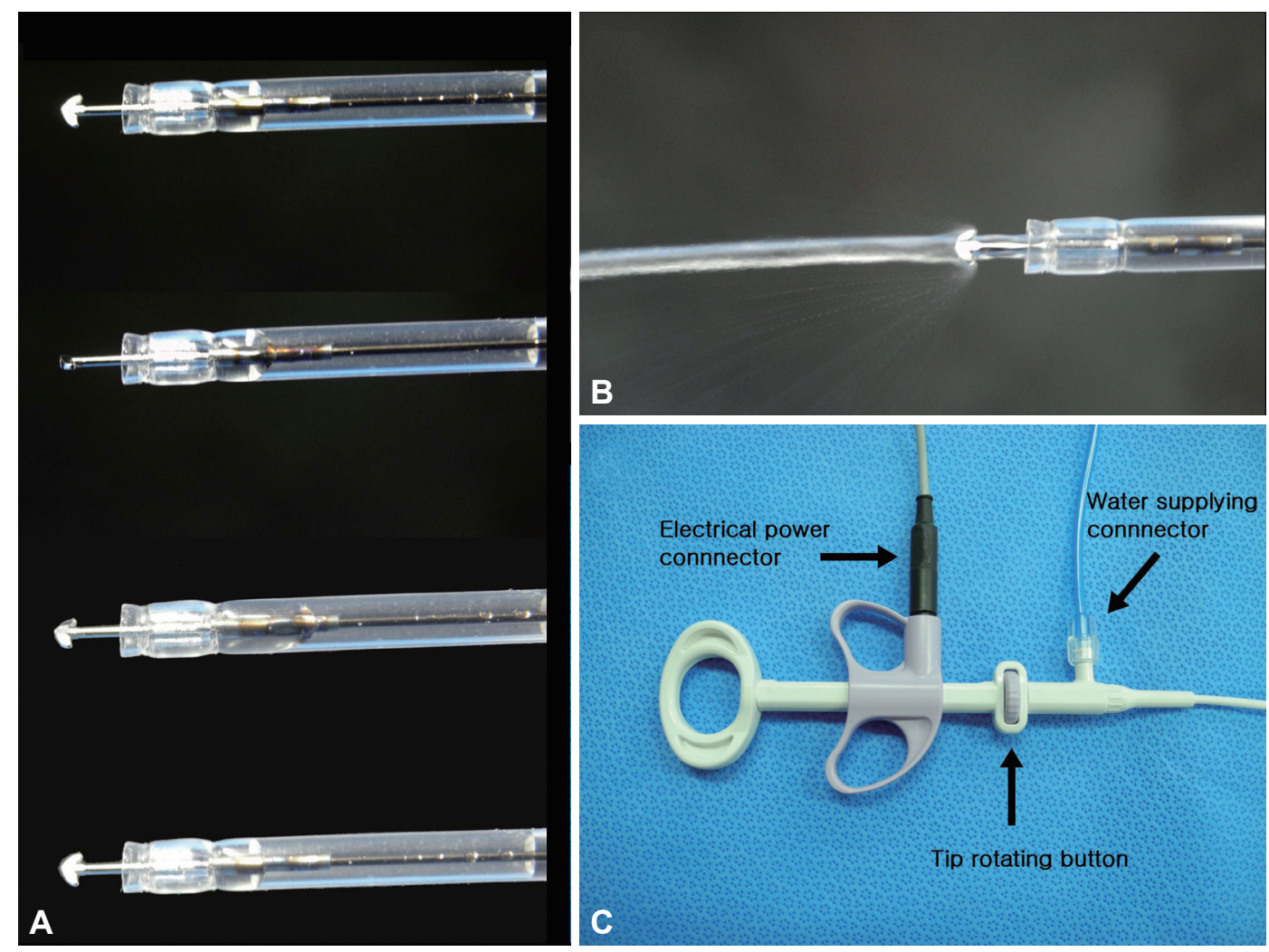

B

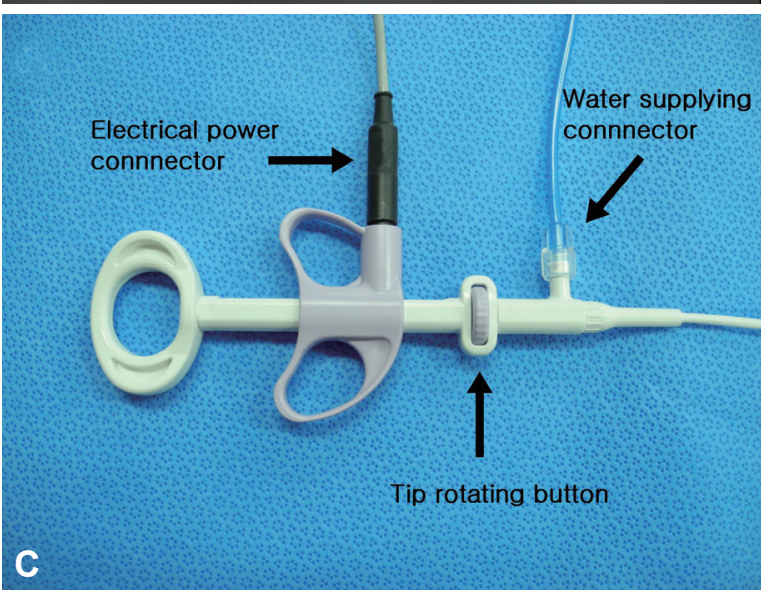

Fig. 1. The novel versatile knife (Optimos knife; Taewoong Medical) used in this study. (A) The Optimos knife has a 1.9-mm-wide and 2.5-mm-long anchor-shaped tip, which can be smoothly and fully rotated by controlling the dial on the handle of the knife. (B) The outer sheath can perform the water injection function. (C) The handle of the knife. 
knife is suitable for obtaining the best visibility during the procedure. Its 1.9-mm-wide, 2.5-mm-long anchor-shaped tip allows a cutting method similar to that by an IT knife or Hook knife, a dissecting method similar to that of a Dual knife or Flex knife, and a hooking method (including vessel coagulation and cutting) similar to that of a Hook knife. The outer sheath enables water irrigation, as with a Flush knife (Fujifilm Medical, Tokyo, Japan) with water-jet function, and allows additional submucosal injection and saline irrigation without the need to change the device. The knife is eligible for a standard endoscope with a working channel width of $\geq 2.8 \mathrm{~mm}$.

\section{Study design}

Two miniature pigs underwent 20 removals of the stomach mucosa, with a target resected specimen size of around $3 \times 3 \mathrm{~cm}$. The stomach of a pig is smaller and anatomically different from that of a human, although the shapes are similar. Heterogeneity between individual pigs also makes it difficult to naturally approach the same scope of mucosa with an endoscope. For this reason, an endoscopic examination was performed before ESD to identify and mark the target areas of the greater curvature of the gastric body at certain intervals, where the endoscope could reach naturally. Then the ESD was repeated using a randomly assigned ESD knife. Completion time of each resection was documented, and the resected specimens were retrieved and evaluated for completeness (en bloc resection).

To assess quality control of the ESD procedures and procedure-related adverse events, detailed histopathological examinations were performed. For examination of short-term adverse events and histopathological changes, the pigs were euthanized 18 hours after the completion of the procedures, and the stomach of each pig was harvested. Gross specimen inspection and detailed histopathological examination and analysis were performed by a pathologist (GIK) who was blinded to the study group.

\section{Devices and settings}

A single-channel endoscope (GIF-Q260J; Olympus Medical Systems Co., Tokyo, Japan) was used for all procedures. A disposable distal attachment cap (D-201; Olympus Medical Systems Co.) was mounted onto the tip of the endoscope. The electrosurgical unit (VIO 300D; ERBE Elektromedizin, Tübingen, Germany) was used as a power source for electrical cutting and coagulation. The settings for all the knives were as follows: (1) soft coagulation, effect 5, $80 \mathrm{~W}$ for marking the target area and effect 3,60 W for vessel coagulation; (2) endocut I, effect 3 (duration 3, interval 3) for marginal incision; and (3) forced coagulation, effect $2,50 \mathrm{~W}$ for submucosal dissection. A 23-gauge endoscopic injection needle (Optimos injector;
Taewoong Medical) was used to inject a mixed solution (a hypertonic saline solution mixed with diluted epinephrine [1: 250,000 ] and slightly stained with indigo carmine). An electric peristaltic pump (Pentax Water Supplier; SA-P2; Hoya Corp., Tokyo, Japan) was used for the water irrigation system of the Optimos knife and the endoscope.

\section{ESD technique}

Two experienced endoscopists (IKC and CIK) performed all procedures (Supplementary Videos 2,3 [available online at http://www.e-ce.org/]); both had performed many ESDs in clinical settings and conducted several teaching programs for ex vivo and in vivo ESD training. All markings on the target mucosa were made circumferentially with the tip of the Optimos knife in the soft coagulation mode and were $2.5 \times 2.5 \mathrm{~cm}$ wide (to make $3 \times 3 \mathrm{~cm}$ wide resected specimens), with 2 to 4 $\mathrm{mm}$ intervals between each marked dot (Fig. 2A).

\section{Optimos knife group}

All ESD steps are shown in Fig. 2. The solution was injected at the distal side of the pseudolesion to achieve an adequate submucosal injection outside the markings. Using a 23-gauge needle, 2 to $5 \mathrm{~mL}$ of the mixed solution was repeatedly injected into the submucosal layer until the target mucosa was completely raised (Fig. 2B). The protruded tip was used to make an incision along the markings, using an endocut mode (Fig. 2CE). The mucosal incision was started at the proximal end of the target lesion and extended around the markings to nearly complete the mucosal incision. In some large lesions, the distal end was saved for maintaining mucosal tension; this method induced easy exposure of the submucosal layer of the proximal target lesion and allowed easier submucosal dissection. Submucosal dissection was gradually carried out using the forced coagulation mode (Fig. 2F-J). Before cutting a vessel, both sides of the vessel were coagulated with the hooking method using the soft coagulation mode until wide discoloration of the vessel was achieved to prevent hemorrhage, and the vessel was cut with the knife using the endocut mode (Fig. 2G-J). Finally, the distal remnant mucosal area was cut with the hooking method (Fig. 2K, L). If bleeding occurred during dissection, saline irrigation using the water injection system of the Optimos knife was used to secure a clear view. The knife in the forced coagulation mode is sufficient to coagulate the small vessels of a ramified vascular network (Fig. 2M-O). The water injection system was also used to keep enough fluid in the submucosa for continuous submucosal dissection (Fig. 2P, Q). The knife was replaced with hemostasis forceps (Coagrasper forceps; Olympus Medical Systems) if initial attempts failed or major bleeding from large blood vessels occurred (Fig. 2R, S). Finally, post-ESD ulcers were observed carefully and large 
vessel stumps were coagulated with Coagrasper forceps in the soft coagulation mode to avoid delayed bleeding (Fig. 2T).

\section{Combination knives group}

Instead of the Optimos knife, three conventional knives (Dual knife, Hook Knife, and IT-2 knife; Olympus Medical Systems Co.) were used for ESD in the combination knives
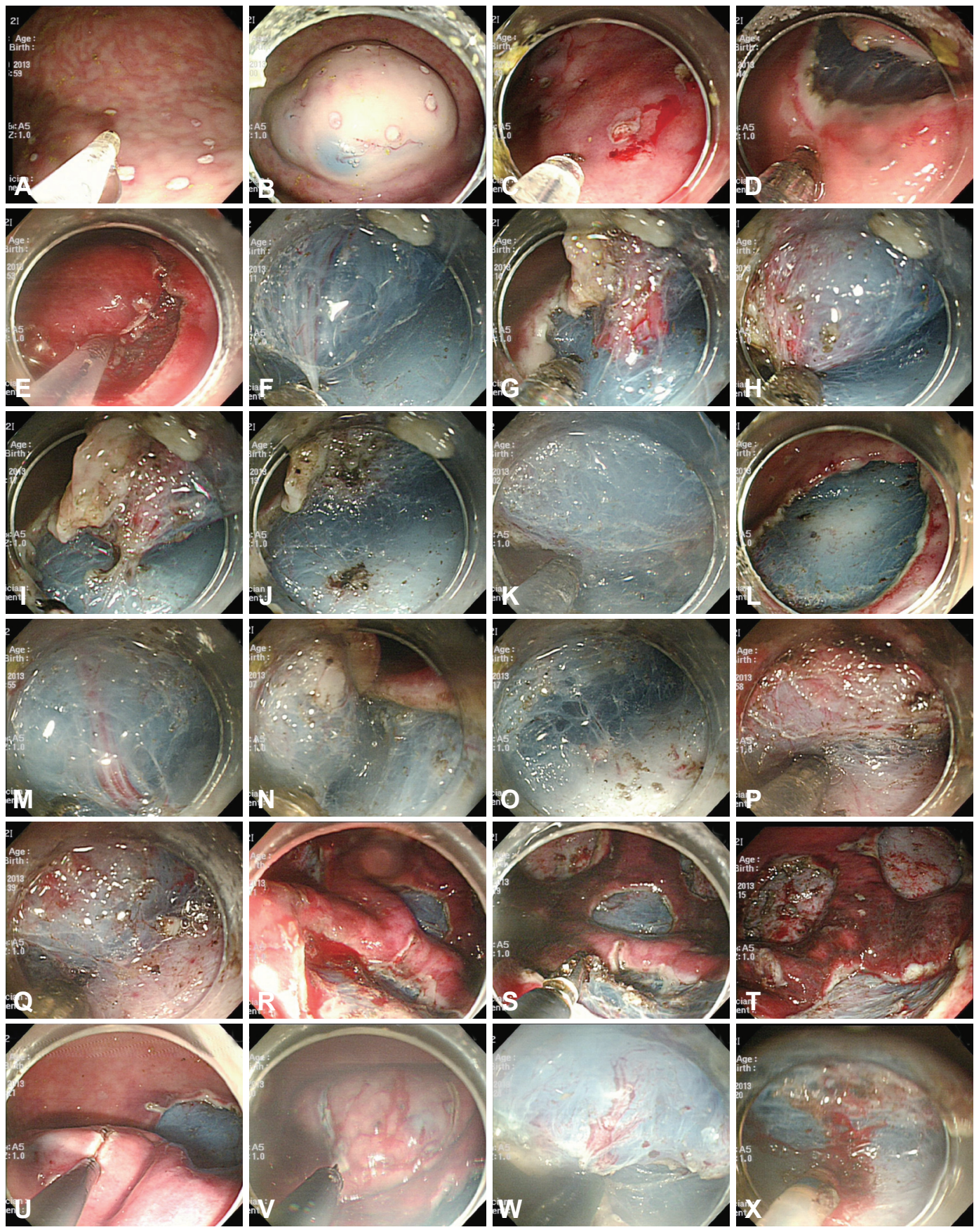

Fig. 2. Endoscopic submucosal dissection (ESD) procedures in the animal model. (A) Markings were made with the tip of the Optimos knife (Taewoong Medical) in the soft coagulation mode. (B) The mixed solution was repeatedly injected into the submucosal layer. (C-E) The tip of the Optimos knife was used to make the incision along the markings, using the endocut mode. (F-J) Submucosal dissection was carried out using the forced coagulation mode. The vessel was coagulated by the hooking method using the soft coagulation mode and cut using the endocut mode. (K, L) The distal remnant mucosal area was cut with the hooking method. (M-O) The knife can coagulate the small vessels of a ramified vascular network in a forced coagulation mode. $(P, Q)$ The water injection system was used to keep enough fluid in the submucosa for continuous submucosal dissection. $(R, S)$ The Coagrasper forceps were used to control major bleeding from large vessels, using the soft coagulation mode. (T) The postESD ulcers were shown to prevent bleeding afterward. (U-X) Three conventional knives (the IT-2, the Hook Knife, and the Dual knife) were used for ESD in the combination knives group. 
group, according to the same steps described for the Optimos knife group. The IT-2 knife was principally used to cut the mucosa and dissect the submucosa. The Dual knife and the Hook knife were used to precut the mucosa or used in difficult circumstances (Fig. 2U-X). To shorten the procedure time, no more than two knives were used.

\section{Endpoint evaluation}

We defined the procedure time as the primary end point. We also assessed completeness of resection, procedural adverse events (bleeding and perforation), and histopathological features.

\section{Procedural measures}

All endoscopic procedures were digitally recorded on a computer. Procedure time was measured from the first injection of the solution to the complete resection of the target area. Cutting speed was measured as the resection area divided by the entire cutting time (duration from start of the mucosal incision to completion of resection). Completeness of resection was assessed by endoscopic and macroscopic inspections of the resection site for any remaining coagulation markings. Significant bleeding was defined as any use of hemostasis. The perforation was diagnosed during endoscopic and histopathological examinations. Procedure time and endpoint events were assessed and recorded by a study assistant not involved in the procedure. Another study assistant took photographs and videos, only when deemed important or critical, so as not to interfere with the procedure time.

\section{Histological evaluation}

After fixing the tissue in formalin, all the endoscopically resected specimens and the remaining stomach were processed. Each resected specimen and stomach was sequentially sectioned and submitted in total for paraffin block embedding and sectioning after staining with hematoxylin and eosin. The fixed specimens were sectioned serially at 2-mm intervals for histological examination. All quantifications of the depth of the lesions in the histological glass slides were performed using the SPOT Advanced program version 5.0 (Diagnostic Instruments, Sterling Heights, MI, USA). The microscopic observations were made based on the following guidelines: the extent of injury to the lamina propria and the submucosa in the resected specimens or the remaining gastric tissue. To evaluate the depth of injury to the muscularis propria (MP), the depth of injury was divided by the whole thickness of the MP layer (inner circular and outer longitudinal), which was expressed as percentage of injury (i.e., when the whole MP was injured, it was expressed as $100 \%$ ). Points showing the most marked or deepest injury were determined and measured.
Histological perforation was considered to be present if there was an injury involving the serosal surface of the remaining gastric tissue.

\section{Statistical analysis}

Mann-Whitney $U$-test was performed for the analysis of the primary objective criteria such as procedure time and specimen area, whereas the chi-square test was used for comparison of adverse events between the two modalities. Independent sample Student $t$-test was also used to compare incidence of injuries between the two modalities. $p<0.05$ was considered significant. Statistical analysis was performed with IBM SPSS version 19.0.0 (IBM Co., Armonk, NY, USA).

\section{RESULTS}

\section{In vivo study}

Two pigs were used for a total of 18 removals of the gastric mucosa (eight removals in the first pig and 10 removals in the second pig; nine removals in the Optimos knife group and nine removals in the combination knives group). Despite the original plan to carry out 10 ESDs per pig, the number of ESDs differed between the two pigs because of the heterogeneity of the approachable scopes, limiting the number of feasible ESDs, as mentioned earlier. All target lesions were concentrated in the greater curvature of the stomach where the endoscope could approach naturally. Since the target lesion became bigger than expected in the first pig, prolonging the procedure time and limiting the number of resections to 8 , we reduced the size of the target lesion in the second pig, thereby shortening the procedure time and allowing 10 resections. The smaller size of the lesion also allowed sufficient submucosal injection before ESD, almost eliminating the need for additional injection during the procedure.

Procedural data are summarized in Table 1, and statistics of each variable are presented in Table 2. All resections were completed as en bloc resections. Procedure time (456 seconds vs. 355 seconds, $p=0.258)$ and cutting speed $\left(1.983 \mathrm{~mm}^{2} / \mathrm{sec}\right.$ vs. $1.57 \mathrm{~mm}^{2} / \mathrm{sec}, p=1.000$ ) were not statistically different. The incidence of bleeding was not significant (1 episode vs. 1 episode). There was no perforation.

\section{Gross inspection}

Both pigs were alive during the 18 hours before euthanasia and necropsy showed no complicated findings. The resected stomachs were incised along the lesser curvature to expose the interior, where no adverse event was found (Fig. 3). All endoscopically resected specimens were rapidly retrieved after the ESD, and pinned onto a rubber board to measure the largest diameter and extent (Fig. 4). The tissues were then placed and 
fixed in $10 \%$ formalin for histological assessment. Hematoxylin and eosin staining was used.

\section{Extent and depth of histological injury}

The extent and depth of tissue injury are summarized in Table 3. The injury to the lamina propria was minimal in all resected specimens. Because the largest extent of injury was only $26 \mu \mathrm{m}$ (10 to $26 \mu \mathrm{m}$; median, 15), the injury did not affect the interpretation of gastric mucosal histology (Fig. 5A, E). The injury to the lamina propria in the remaining gastric bed tissue was more extensive ( 2 to $7.3 \mathrm{~mm}$; median, 3.5) than that in the resected specimen because the stomach was harvested 18 hours after the procedures (Fig. 5B, F). The difference of lamina propria injury both in the ESD specimen and the remaining bed tissue was not significant between the two groups $(p=0.800$ and $p=0.720$, respectively) (Fig. $6 \mathrm{~A}, \mathrm{~B}$ ). The extent of injury to the submucosa in the resected specimens ranged from 56 to $668 \mu \mathrm{m}$ (median, 239.5), whereas the injury did not affect the quality of the lamina propria (Fig. 5C, G). There was no significant difference between the two modalities ( $p=0.483$ ) (Fig. $6 \mathrm{C})$. The incidence of injury to the MP ranged from $0 \%$ to $20.1 \%$ (median, 8.4) compared to that in the entire normal

Table 1. Procedural Data of All 18 Endoscopic Submucosal Dissection Procedures

\begin{tabular}{|c|c|c|c|c|c|c|c|c|c|c|c|}
\hline No./Dr & $\begin{array}{l}\text { No. of } \\
\text { mini } \\
\text { pigs }\end{array}$ & Knife & $\begin{array}{c}\text { Resection } \\
\text { diameter, } \\
\text { mm }\end{array}$ & $\begin{array}{c}\text { Resection } \\
\text { square, } \\
\mathrm{mm}^{2}\end{array}$ & $\begin{array}{l}\text { Procedure } \\
\text { time, } \\
\text { sec }\end{array}$ & $\begin{array}{l}\text { Injection } \\
\text { time, } \\
\text { sec }\end{array}$ & $\begin{array}{l}\text { Cutting } \\
\text { time, } \\
\text { sec }\end{array}$ & $\begin{array}{l}\text { Cutting } \\
\text { speed, } \\
\mathrm{mm}^{2} / \mathrm{sec}\end{array}$ & $\begin{array}{c}\text { Complete- } \\
\text { ness }\end{array}$ & $\begin{array}{l}\text { Bleeding } \\
\text { frequency }\end{array}$ & Perforation \\
\hline $1 / \mathrm{CK}$ & 1 & Opt & $30 \times 30$ & 900 & 786 & 75 & 711 & 1.266 & Yes & 0 & 0 \\
\hline $2 / \mathrm{CK}$ & 1 & Comb & $32 \times 30$ & 960 & 867 & 84 & 783 & 1.226 & Yes & 0 & 0 \\
\hline 3/IC & 1 & Opt & $40 \times 38$ & 1,520 & 1,041 & 122 & 919 & 1.654 & Yes & 0 & 0 \\
\hline 4/IC & 1 & Comb & $30 \times 23$ & 690 & 709 & 105 & 604 & 1.142 & Yes & 0 & 0 \\
\hline $5 / \mathrm{CK}$ & 1 & Comb & $28 \times 23$ & 644 & 343 & 107 & 236 & 2.729 & Yes & 0 & 0 \\
\hline 6/CK & 1 & Opt & $30 \times 25$ & 750 & 1,145 & 100 & 1,045 & 0.718 & Yes & 0 & 0 \\
\hline 7/IC & 1 & Opt & $40 \times 34$ & 1,360 & 1546 & 163 & 1,383 & 0.983 & Yes & 1 & 0 \\
\hline 8/IC & 1 & Comb & $25 \times 23$ & 575 & 320 & 75 & 245 & 2.347 & Yes & 0 & 0 \\
\hline 9/CK & 2 & Opt & $30 \times 30$ & 900 & 429 & 99 & 330 & 2.727 & Yes & 0 & 0 \\
\hline 10/CK & 2 & Opt & $26 \times 26$ & 676 & 396 & 100 & 296 & 2.284 & Yes & 0 & 0 \\
\hline 11/IC & 2 & Opt & $30 \times 30$ & 900 & 340 & 91 & 249 & 3.614 & Yes & 0 & 0 \\
\hline 12/IC & 2 & Opt & $28 \times 25$ & 700 & 456 & 103 & 353 & 1.983 & Yes & 0 & 0 \\
\hline 13/CK & 2 & Comb & $30 \times 28$ & 840 & 665 & 130 & 535 & 1.570 & Yes & 0 & 0 \\
\hline 14/CK & 2 & Comb & $25 \times 24$ & 600 & 274 & 50 & 224 & 2.679 & Yes & 0 & 0 \\
\hline 15/IC & 2 & Opt & $25 \times 22$ & 550 & 277 & 55 & 222 & 2.477 & Yes & 0 & 0 \\
\hline 16/IC & 2 & Comb & $22 \times 20$ & 440 & 339 & 52 & 287 & 1.533 & Yes & 1 & 0 \\
\hline 17/CK & 2 & Comb & $32 \times 30$ & 960 & 355 & 68 & 287 & 3.345 & Yes & 0 & 0 \\
\hline 18/CK & 2 & Comb & $20 \times 20$ & 400 & 497 & 96 & 401 & 0.998 & Yes & 0 & 0 \\
\hline
\end{tabular}

Comb, combination knives; Opt, optimos knife.

Table 2. Statistical Analysis of Procedural Data

\begin{tabular}{|c|c|c|c|}
\hline Variable & Optimos knife, median (IQR) & Combination knives, median (IQR) & $p$-value \\
\hline Procedure time, sec & $456(645)$ & $355(326)$ & 0.258 \\
\hline Injection time, sec & $100(14)$ & $84(37)$ & 0.340 \\
\hline Cutting time, sec & $353(623)$ & $287(290)$ & 0.297 \\
\hline Resection square, $\mathrm{mm}^{2}$ & $900(684)$ & $644(115)$ & 0.136 \\
\hline Cutting speed, $\mathrm{mm}^{2} / \mathrm{sec}$ & $1.983(1,211)$ & $1.57(1,453)$ & 1.000 \\
\hline Completeness rate (en bloc resection rate), \% ( $n /$ total) & $100(9 / 9)$ & $100(9 / 9)$ & \\
\hline Bleeding rate, $\%$ ( $n /$ total) & $10(1 / 9)$ & $10(1 / 9)$ & 1.000 \\
\hline Perforation rate, $\%$ ( $n /$ total) & $0(0 / 9)$ & $0(0 / 9)$ & \\
\hline
\end{tabular}

Values are analyzed using the Mann-Whitney $U$-test.

IQR, interquantile range. 
muscular layer (Fig. 5D, H). No significant difference in the incidence of injury to the MP was found between modalities $(p=0.166)$ (Fig. 6D).

\section{DISCUSSION}

We have reported herein the results of an animal feasibility study for ESD using the Optimos knife, which was developed by combining the advantages of various existing knives. We summarize the aforementioned features of the Optimos knife as follows: it is capable of performing several steps of ESD, has a smoothly rotating tip, transparent outer sheath, and water injection function. The distinguishable features among the recently developed knives are water injection function

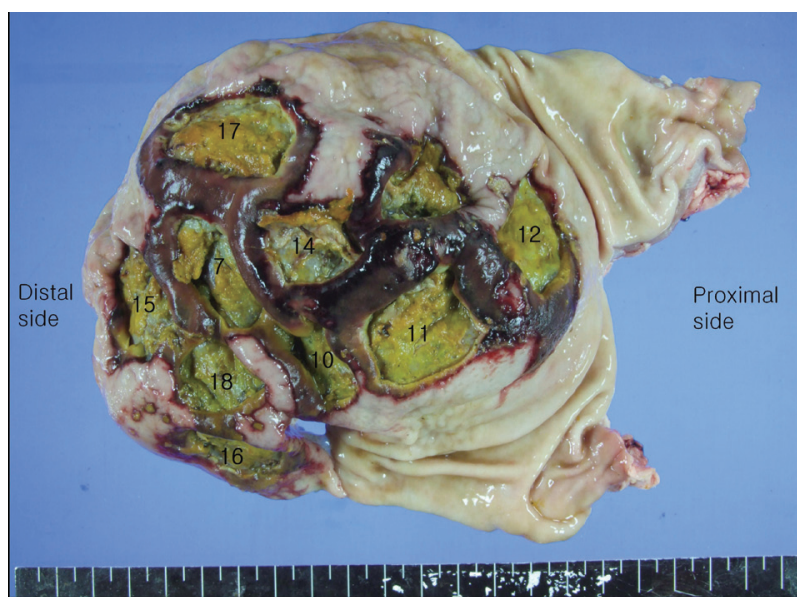

Fig. 3. Part of the stomach harvested from the second pig. Target lesions were centered on the greater curvature of the gastric body. The resected stomachs were incised along the lesser curvature to expose the interior, where no abnormality such as bleeding or perforation was found. The surrounding normal tissues were damaged and changed, but were not detected during endoscopic submucosal dissection. All numbers represent sequentially resected areas.

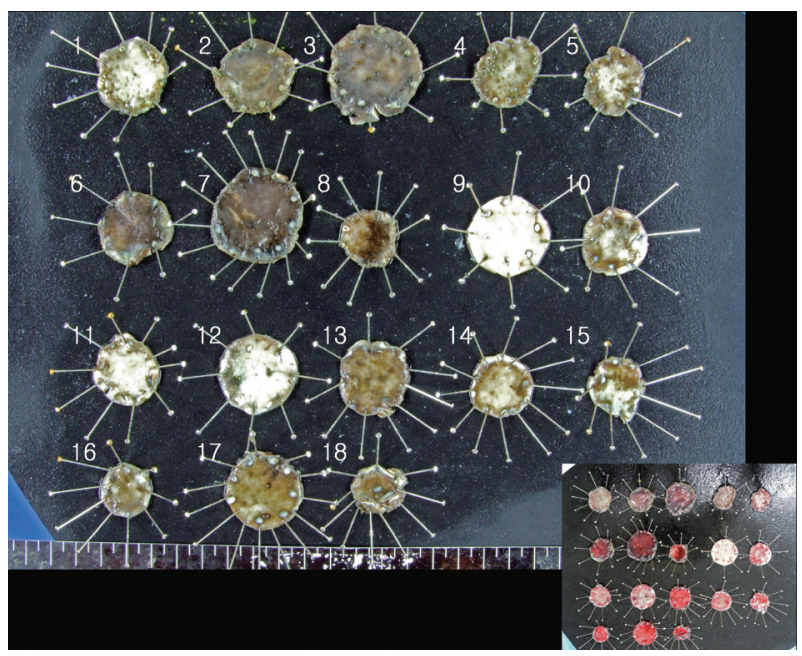

Fig. 4. Tissues from resected specimens pinned on a rubber board after formalin fixation. (Inset) Resected specimens just after the procedure. and hemostatic capability for quality-controlled ESD. ${ }^{16-18}$ Water injection function, first adopted in the Flush knife, allows safer and faster ESD by decreasing the number of submucosal injections, which have to be repeated many times during ESD. ${ }^{19}$ In addition, the ball-type Flush knife, like the Dual knife, was reported to show good results due to enhanced hemostatic capability. ${ }^{16}$ The Hook knife is widely used for the advanced ESD technique, allowing free redirection of the tip for the hooking method; however, since there is no dial for rotation, the whole handle has to be rotated for redirection, limiting delicate and accurate redirection.

The limited outer sheath diameter makes it technically difficult to combine all these advantages in one knife. An outer sheath where a knife can be safely inserted, an anchor-shaped smoothly and fully rotating tip, and an outer sheath for the water injection function, all have to be contained in a limited diameter, and at an affordable cost, which is very difficult.

This animal feasibility study was performed to compare the performance and safety of the Optimos knife, which was developed to satisfy all the aforementioned features, with those of the widely used, conventional knives. While planning the study, we expected that the procedure time would be longer in the combination knives group, where the knives have to be replaced frequently. Procedure time and cutting speed in animal studies are mostly affected by the location and size of target lesions, and adverse events. ${ }^{17}$ Our results show that procedure time and cutting speed were not different between the two modalities, which may be significant, since a longer procedure time may be expected in the Optimos knife group given the relatively but not statistically bigger resection width in this group. The overall results were not satisfactory, probably due to the following factors: the procedure was performed safely and within a very short period of time by experienced endoscopists who were also experienced in in vivo ESD; the procedure was performed at easier, not difficult, locations; target lesions were relatively small; and an initial submucosal injection before ESD was sufficient to eliminate the need for the water injection system. The number of specimens was below the expected number because many target lesions had to be resected in two miniature pigs, and resections of various sizes were made in an attempt to prevent any effect on other lesions in the vicinity within a limited space. Accessories were replaced very quickly by skilled assistants. ESD was also performed for targeted normal lesions, where the difficulty was not more than that for pathological lesions. The stomachs of the pigs were very thick with relatively small blood vessels, almost eliminating the risk of perforation or bleeding. In addition, being an animal study, not every procedure was accompanied by preliminary coagulation of the exposed vessels to prevent bleeding. Since the anatomical shape of the stomach 
Table 3. Extent or Depth of Histological Injury in the Lamina Propria, Submucosa, and Muscularis Propria

\begin{tabular}{|c|c|c|c|c|c|c|}
\hline No. & Knife & LP injury in ESD, $\mu \mathrm{m}$ & LP injury in bed, mm & SM injury in ESD, $\mu \mathrm{m}$ & MP injury in bed, \% & Perforation \\
\hline 1 & Optimos knife & 10 & 2.2 & 352 & 0 & No \\
\hline 2 & Combination knives & 26 & 7.3 & 623 & 15.3 & No \\
\hline 3 & Optimos knife & 15 & 3.6 & 225 & 12.5 & No \\
\hline 4 & Combination knives & 15 & 2.5 & 254 & 18.5 & No \\
\hline 5 & Combination knives & 15 & 2 & 122 & 0 & No \\
\hline 6 & Optimos knife & 20 & 5.4 & 655 & 0 & No \\
\hline 7 & Optimos knife & 12 & 2.5 & 330 & 8.1 & No \\
\hline 8 & Combination knives & 17 & 4.3 & 264 & 16.2 & No \\
\hline 9 & Optimos knife & 10 & 6.2 & 222 & 9.8 & No \\
\hline 10 & Optimos knife & 18 & 3.8 & 189 & 5 & No \\
\hline 11 & Optimos knife & 19 & 4.4 & 212 & 0 & No \\
\hline 12 & Optimos knife & 18 & 2.1 & 56 & 5.8 & No \\
\hline 13 & Combination knives & 13 & 5.2 & 772 & 5.6 & No \\
\hline 14 & Combination knives & 13 & 2.7 & 356 & 0 & No \\
\hline 15 & Optimos knife & 10 & 2.3 & 88 & 11.9 & No \\
\hline 16 & Combination knives & 18 & 5.1 & 110 & 8.7 & No \\
\hline 17 & Combination knives & 10 & 2.6 & 440 & 20.1 & No \\
\hline 18 & Combination knives & 10 & 3.3 & 92 & 12.4 & No \\
\hline
\end{tabular}

LP, lamina propria; ESD, endoscopic submucosal dissection; SM, submucosa; MP, muscularis propria.
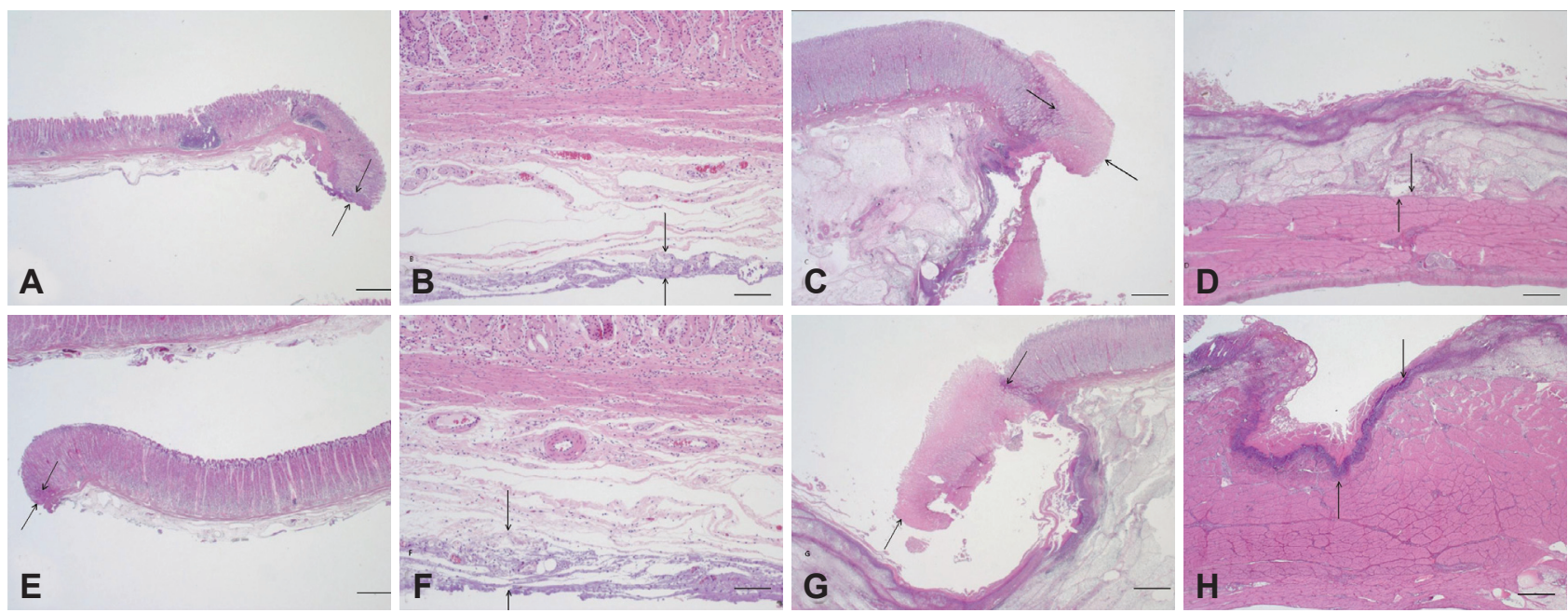

Fig. 5. Representative histological findings of endoscopic mucosal dissection (ESD) specimens and residual muscularis propria tissue from the Optimos knife group (A-D) and combination knives group (E-H; H\&E stain). (A, B) ESD showing minimal injury to the lamina propria and submucosa in the Optimos knife group. (C, D) Residual bed tissue showing minimal injury to the lamina propria and muscularis propria in the Optimos knife group. (E, F) ESD showing minimal injury to the lamina propria and submucosa in the combination knives group. (G, H) Residual bed tissue showing marked injury in the lamina propria and muscularis propria in the combination knives group. Arrows represent the extent of injury. Scale bars represent $50 \mu \mathrm{m}$ in $(B)$ and $(F)$ and $500 \mu \mathrm{m}$ in the others.

of the miniature pigs was different from that of humans, there were some areas that were not as easily approachable as first expected, which could have become another factor for bias.

To distinguish this study from previously reported animal studies using new knives, we performed more detailed histopathological analyses including the evaluation of direct tissue injury related to ESD, although not for determining the oc- currence of adverse events. Previous studies, which included necropsy and histopathological analysis of the remaining bed tissues immediately after ESD, could not find a difference between the resected specimen and the remaining bed tissue, when assessing micro-injury to the tissue by a knife. . $^{17,18,20,21}$ On the other hand, we performed a necropsy after a short interval, for more accurate analysis. We anticipated that, in this 


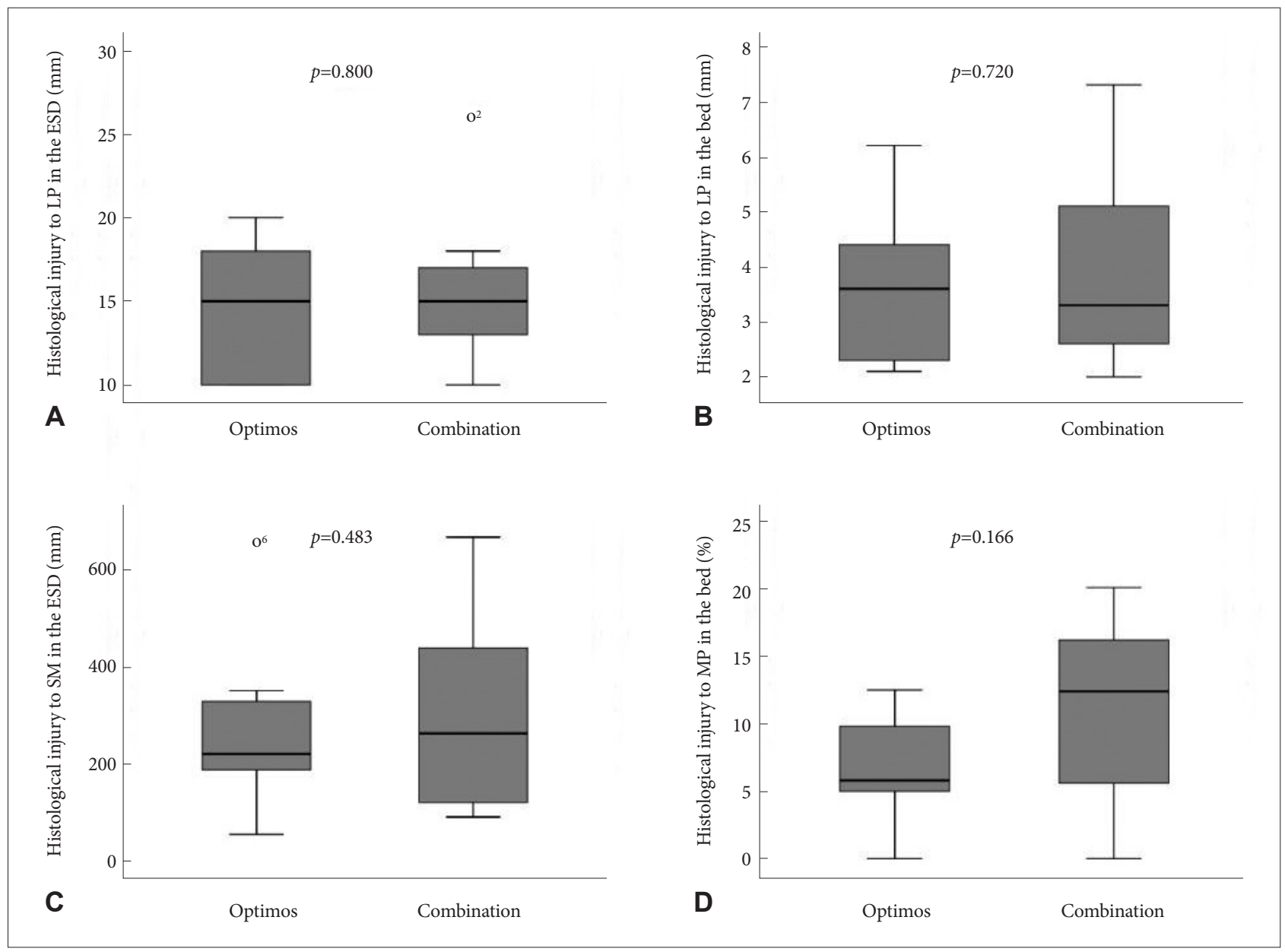

Fig. 6. Comparison of the levels of histological injury between the Optimos knife group and the combination knives group. (A) Extent of injury to the lamina propria in the dissected gastric specimens. (B) Extent of injury to the lamina propria in the remaining gastric bed tissue. (C) Depth of injury to the submucosa in the dissected gastric specimens. (D) Depth of injury to the MP in the remaining gastric bed tissue. LP, lamina propria; ESD, endoscopic mucosal dissection; SM, submucosa; MP, muscularis propria.

method, we could accurately evaluate the occurrence of adverse events such as bleeding or perforation and the extent of tissue injury. The resected target specimens were immediately fixed in formalin for histopathological analysis, whereas necropsy was performed 18 hours later for evaluation of tissue injury progression. Therefore, the resected stomachs were harvested and a separate histopathological analysis was performed for the remaining bed tissues. As seen in Fig. 3, the surrounding normal tissues were found to be damaged and changed, but were not identified during ESD. However, the degrees of damage were not different between the two groups. There were also differences in the remaining bed tissues obtained from the same as well as different sources, indicating the presence of complex underlying causes such as the lack of proper blood supply due to cut vessels during ESD, injury from excessive injection of hypertonic saline, or more severe procedure-related injury due to the short interval between target areas, rather than the direct influence of the knives.

Although our study could not confirm qualitative superi- ority of the Optimos knife over other knives in terms of procedure time or various aspects of ESD, this study looked at the technical features of the Optimos knife, and is a meaningful noninferiority test that compared the function as well as the safety of the Optimos knife with those of the existing knives. We believe that determining the difference can be difficult when the existing accessories are good and endoscopists and assistants are skilled. The Optimos knife seems convenient to use while performing quality-controlled ESD and has various advantages that makes it a better option: (1) the Optimos knife allows direct visualization of the lesion for cutting or dissection, like the Dual knife or the Flex knife, and the transparent outer sheath offers clear visibility of the lesion; (2) the hooking method is available for cutting or dissection, similar to the IT-2 knife or the Hook knife, in addition to hemostatic capability for direct coagulation of blood vessels; and (3) as in the Hook knife, the tip can be fully and smoothly rotated by controlling the dial on the handle of the knife; and fourth, as in the Flush knife, the Optimos knife is equipped with a water 
injection system to ensure a safe and rapid procedure by reducing the number of submucosal injections that have to be repeated many times during the ESD procedure. Most of all, it is noteworthy that the basic three steps, including marking, incision, and submucosal dissection, can be achieved with only one knife, which is important in terms of cost reduction, insurance coverage, procedural simplicity, and safety.

This study has several limitations, including the lack of sufficient ESD specimens due to small sample size, non-normal distribution of data and target lesions, execution of procedures by experienced endoscopists, lack of bigger and variously located target lesions, short interval between target lesions that affect the surrounding tissues, and use of universal electrical settings for all knives. Due to the inherent limitations of an animal study, the procedures were performed at small sites during a certain period in multiple procedures were performed in each pig, making the target size of each specimen relatively small and rather insufficient to observe results that could highlight the advantages of this new knife. A prospective, comparative study involving larger lesions and a greater sample size is required to better observe the advantages of the Optimos knife. A head-to-head comparison with each of the conventional knives would provide more objective data.

In conclusion, this study could not confirm qualitative superiority of the Optimos knife over other knives in terms of procedure time or various aspects of ESD, but this study investigated the technical features of the Optimos knife and is meaningful as a noninferiority test of function as well as safety of the Optimos knife compared to the existing knives. Further large-scaled, prospective clinical studies are required for more accurate determination of its safety and efficacy.

\section{Conflicts of Interest}

Chang-Il Kwon invented the Optimos knife in conjunction with Taewoong Medical Company.

\section{Acknowledgments}

This study was supported by a grant of the GMEDECA Center R\&D Project (KERI 12-30-I0199-03), Gyeonggi Provincial Government, the Republic of Korea; by grants from the SNUBH Research Fund (02-2007-027); and also by the National Center of Efficacy Evaluation for the Development of Health Products Targeting Digestive Disorders (NCEED). We wish to thank Dr. Takashi Toyonaga and Dr. Shinwa Tanaka (Department of Endoscopy, Kobe University, Kobe, Japan) for technical advice on the water injection system. We also thank Woo Jin Shin, Jong Hoon Rim, and Kil Soo Kim for their kind assistance in all procedures.

\section{SUPPLEMENTARY MATERIAL}

Video 1. The novel versatile knife (Optimos knife) used in this study. The tip of the knife can be rotated fully and smoothly by controlling the button on the handle. The transparent sheath of the knife is suitable for attaining the best view during the procedure. The 1.9-mm-wide and 2.5-mm-long anchor-shaped tip allows the performance of several steps of ESD including marking, circumferential incision and cutting, and dissection and vessel coagulation. The outer sheath allows additional submucosal injection and saline irrigation without changing the device.

Videos 2, 3. Endoscopic submucosal dissection procedures in the animal model. All markings on the target mucosa were circumferentially made with the tip of the Optimos knife, using a soft coagulation mode. Using a 23-gauge needle, the mixed solution was repeatedly injected into the submucosal layer until the target mucosa was totally raised. In the Optimos knife group, mucosal incision was started at the proximal end of the target lesion and extended around the markings using an endocut mode to almost complete the mucosal incision. Submucosal dissection was gradually carried out, using the forced coagulation mode. Finally, the distal remnant mucosal area was cut with the hooking method. If bleeding occurs during dissection, saline irrigation can be performed for securing a clear view using the water injection system of the Optimos knife. The knife in a forced coagulation mode is sufficient to coagulate the small vessels of a ramified vascular network. The water injection system was also used to keep enough fluid in the submucosa for continuous submucosal dissection. In the combination knives group, three conventional knives (the IT-2 knife, the Hook Knife, and the Dual knife) were used according to the same steps as the Optimos knife group.

\section{REFERENCES}

1. Ono H, Kondo H, Gotoda T, et al. Endoscopic mucosal resection for treatment of early gastric cancer. Gut 2001;48:225-229.

2. Ohkuwa M, Hosokawa K, Boku N, Ohtu A, Tajiri H, Yoshida S. New endoscopic treatment for intramucosal gastric tumors using an insulated-tip diathermic knife. Endoscopy 2001;33:221-226.

3. Yamamoto H, Kawata H, Sunada K, et al. Success rate of curative endoscopic mucosal resection with circumferential mucosal incision assisted by submucosal injection of sodium hyaluronate. Gastrointest Endosc 2002;56:507-512.

4. Miyamoto S, Muto M, Hamamoto Y, et al. A new technique for endoscopic mucosal resection with an insulated-tip electrosurgical knife improves the completeness of resection of intramucosal gastric neoplasms. Gastrointest Endosc 2002;55:576-581.

5. Chung IK, Lee JH, Lee SH, et al. Therapeutic outcomes in 1000 cases of endoscopic submucosal dissection for early gastric neoplasms: Korean ESD Study Group multicenter study. Gastrointest Endosc 2009;69: 1228-1235.

6. Yahagi N, Fujishiro M, Kakushima N, et al. Endoscopic submucosal dissection for early gastric cancer using the tip of an electrosurgical snare (thin type). Dig Endosc 2004;16:34-38.

7. Isomoto H, Shikuwa S, Yamaguchi N, et al. Endoscopic submucosal dissection for early gastric cancer: a large-scale feasibility study. Gut 2009;58:331-336.

8. Watanabe K, Ogata S, Kawazoe S, et al. Clinical outcomes of EMR for gastric tumors: historical pilot evaluation between endoscopic submucosal dissection and conventional mucosal resection. Gastrointest Endosc 2006;63:776-782.

9. Toyonaga T, Nishino E, Man IM, East JE, Azuma T. Principles of quality controlled endoscopic submucosal dissection with appropriate dissection level and high quality resected specimen. Clin Endosc 2012;45: 362-374.

10. Cao Y, Liao C, Tan A, Gao Y, Mo Z, Gao F. Meta-analysis of endoscopic submucosal dissection versus endoscopic mucosal resection for tumors of the gastrointestinal tract. Endoscopy 2009;41:751-757.

11. Yamamoto H, Kita H. Endoscopic therapy of early gastric cancer. Best Pract Res Clin Gastroenterol 2005;19:909-926.

12. Kakushima N, Fujishiro M. Endoscopic submucosal dissection for gastrointestinal neoplasms. World J Gastroenterol 2008;14:2962-2967.

13. Conlin A, Kaltenbach T, Kusano C, Matsuda T, Oda I, Gotoda T. Endoscopic resection of gastrointestinal lesions: advancement in the ap- 
plication of endoscopic submucosal dissection. J Gastroenterol Hepatol 2010;25:1348-1357.

14. Oyama T. Counter traction makes endoscopic submucosal dissection easier. Clin Endosc 2012;45:375-378.

15. Neuhaus H. Endoscopic submucosal dissection in the upper gastrointestinal tract: present and future view of Europe. Dig Endosc 2009;21 Suppl 1:S4-S6.

16. Toyonaga T, Man IM, Fujita T, et al. The performance of a novel balltipped Flush knife for endoscopic submucosal dissection: a case-control study. Aliment Pharmacol Ther 2010;32:908-915.

17. Fujishiro M, Sugita N. Animal feasibility study of an innovated splashneedle for endoscopic submucosal dissection in the upper gastrointestinal tract. Dig Endosc 2013;25:7-12.

18. Fukami N, Ryu CB, Said S, Weber Z, Chen YK. Prospective, random- ized study of conventional versus HybridKnife endoscopic submucosal dissection methods for the esophagus: an animal study. Gastrointest Endosc 2011;73:1246-1253.

19. Toyanaga T, Man IM, Ivanov D, et al. The results and limitations of endoscopic submucosal dissection for colorectal tumors. Acta Chir Iugosl 2008;55:17-23.

20. von Renteln D, Pohl H, Vassiliou MC, Walton MM, Rothstein RI. Endoscopic submucosal dissection by using a flexible Maryland dissector: a randomized, controlled, porcine study (with videos). Gastrointest Endosc 2010;71:1056-1062.

21. Neuhaus H, Wirths K, Schenk M, Enderle MD, Schumacher B. Randomized controlled study of EMR versus endoscopic submucosal dissection with a water-jet hybrid-knife of esophageal lesions in a porcine model. Gastrointest Endosc 2009;70:112-120. 